Phone: (+01) 6106824146

Email: Info@TEAsystems.com

http://www.TEAsystems.com

TEA Systems Corporation

65 Schlossburg St.

Alburtis, PA 18011 USA

\title{
Methods for comparative extraction of OPC response
}

\author{
Terrence E. Zavecz - TEA Systems \\ tzavecz@TEAsystems.com \\ Phone: (+01) 6106824146
}

Copyright 2007, Society of Photo-Optical Instrumentation Engineers. And TEA Systems Corp.

This paper was published in SPIE Vol 6520-64 and is made available as an electronic reprint or preprint with permission of SPIE. One print or electronic copy may be made for personal use only. Systematic or multiple reproduction, distribution to multiple locations via electronic or other means, duplication of any material in this paper for a fee or for commercial purposes or modification of the content of the paper are prohibited. 


\title{
Methods for comparative extraction of OPC response
}

\author{
Terrence E. Zavecz \\ TEA Systems, 65 Schlossburg St., Alburtis, PA 18011 USA; tzavecz@TEAsystems.com
}

\begin{abstract}
1. ABSTRACT
The current ITRS roadmap details the growing complexity of device design and the latest device-manufacturer's techniques for tuning their process for each new design generation. In spite of the current desire to incorporate techniques termed "Design for Manufacture" (DFM) into the sequence, simulations and the design cycle do little more than optimize feature quality for ideal exposure conditions while testing for shorts, opens and overlay problems over process variations. Testing in the DFM simulation is performed by the adaptation of a technique unchanged in the last 30 years, the Process Window analysis. With this, mediocre successes seen in chip-design have not taken their share of the burden of technology advancement. Consequently, process adaptation to each new design has fallen to increasingly complex setup procedures of the exposure toolsets that customize scanner performance for each new device.
\end{abstract}

Design optimization by simulation focuses on feature layout optimization for resolution. Design solutions that take advantage of the full potential spectrum of mask-feature alternatives to increase functional process-space and simplify setup in manufacturing do not exist since there is no method of feedback. A mechanism is needed that can quantify design performance robustness, with mask-contributions, to variations in the user's specific manufacturing process.

In this study, a Process Behavior Model methodology is presented for the analysis of feature profiles and films to derive the relative robustness of response to process variations for alternative OPC designs. Analysis is performed without regard to the specific mechanics of the design itself. The design alternatives of each OPC feature are shown to be strong contributors not only to resolution and depth-of-focus but also to the stability of final image response; that is the ability of the feature profile to remain at optimum under varying conditions of process exposure excursion.

Several different, $70 \mathrm{~nm}$ multi-pitch OPC designs are compared for their response stability to fluctuations of the process. The optimal process corrections on the reticle are shown to be dependent upon not only the final image size at some optimal exposure point but also on the ability of the design to maintain feature size within tolerance across an increasingly large process-space of the target production process. The failure of the classic Process Window analysis to anticipate or provide corrective insight for performance improvement under these conditions is illustrated.

Models are presented that allow the extraction of the nonlinear but systematic interactions of several OPC designs with the normal fluctuations experienced across the process exposure space plus those introduced by the toolset and film-stack variation. A method of extracting the systematic component of each feature's design-iteration is derived providing the ability to quantify the specific OPC response sensitivity to changes in the exposure and process films as well as drift introduced by the tools of the exposure set.

Keywords: Lithographic Friendly Design, DFM, Bossung, Dose Control, Double Patterning, Dose Uniformity, ACLV, Process Behavioral Models, metrology, stability, matching, photomask, simulation, proximity, response

\section{MOTIVATION AND INTRODUCTION}

\section{Interactions between Design and Lithography}

The ITRS roadmap for 2005, in outlining the path to sub $65 \mathrm{~nm}$ MPU half-pitch production, highlighted the need for "Litho friendly design rules" as it coupled the path with some very complex process and product design sequences needed to achieve these goals. ${ }^{i}$ The design segments of our industry presented the need for improved simulation accuracy coupled with a need for chip-designs that consider the capabilities of the production process. Production complexity continues to increase with the major burden of the solutions falling into the realm of the scanner vendors. Production complexity grows rapidly in this segment with the introduction of techniques that customize exposure tool setup to the characteristics of the specific chip-design. Customization efforts here introduced techniques such as aberration monitoring, source configuration, and active adjustment of the exposure dose first across the wafer and then within the exposure field itself. These are 
techniques that involve both pattern and process specific pre-exposure setup. They are time-consuming, capacity-lowering and results are not fully verifiable by the user until production begins.

While feature resolution in low contrast processes, typically called "low $\mathrm{k} 1$ ", is always a problem, the most serious limitations currently facing the industry are strongly embedded in the complexity in mask making, pattern design and highdensity feature processing. Low k1 manufacturing solutions continue to be applied solely at the process level with their scope limited to the process characteristics of the specific design. For example it's been shown that process disturbances vary across the wafer in a highly systematic manner that can be modeled based upon either the features location on the wafer or upon the exposure field. ${ }^{\text {ii }}$ Systematic variations across the exposure field caused by design or mask process are therefore corrected using the setup ability of the exposure tool to vary exposure-dose with location. Additional dosemapping is anticipated to improve the uniformity of CD's across the substrate to optimize bake-plate impact. The process is therefore tuned to the specific photomask and the individual design's sensitivity to process fluctuations.

Because of the complexity of lithographic interactions between process and design, the in-process hopes of expanding the functional exposure process space by using an Automated Process Control (APC) solution have not matured beyond the handling of overlay offsets from lot-to-lot and the integration of in-line metrology for wafer-gating using statistical Critical Dimension (CD) and overlay control.

The solution-set visualized in the roadmap thus addresses the associated production needs for DRAM CD control of 6.6 nanometers (nm), dropping down to $4.2 \mathrm{~nm}$ by 2011 and with an associated overlay tolerance of $8.1 \mathrm{~nm}$, by using techniques that customize the process to each specific design and mask level. These solutions continue to increase the complexity of per-design setup to the point that additional specialized exposure tool setup packages involving lengthy offline testing will be needed to continue on this path. Even with these complex techniques, recent problems with the nextgeneration development of Extreme Ultra Violet (EUV) lens materials and manufacturing techniques have threatened to delay the attainment of these goals unless the industry deviates from the customary methods used for manufacture. In search of a solution, recent papers have suggested a need to split up the most-dense pattern layers using a technique called "Double Patterning". iii

Double Patterning essentially splits the densest, critical layers of a device thereby reducing the proximity-resolution problems arising from scatter and aberration compensation. In doing so, it increases the load on overlay control without reducing setup complexity. The adaptation of this technique will add to the already high number of mask layers projected for production to levels beyond the 36 MPU levels currently envisioned for $32 \mathrm{~nm}$ half-pitch production.

The semiconductor industry has moved the major burden of improving manufacturing stability and yield from control in the design realm and onto the shoulders of the process equipment vendors. Success or failure of each design relies heavily on the vendor's ability to tune its toolset to the specific characteristics of each unique design and critical layer mask. As a result the new industry roadmaps will entail higher production costs and lower startup yields as technical progress continues unless it includes a true method of incorporating manufacturability into the "Litho-Friendly" design sequence.

\section{Current Problems in Lithographic Friendly Design}

The current approach to Lithographic Friendly Designs involves the use of process simulators that employ fundamental photoresist and wavefront models in an attempt to predict the final image quality of mask images that incorporate nonprinting, sub-resolution patterns or phase shifting features. These non-printing features are employed in an attempt to locally modify the wavefront, forcing it to print the high-density, proximity sensitive features of today's devices. ${ }^{\text {iv }}$

Simulations have historically focused only on the few, critical representative features of a layer for initial process setup. Software-based wavefront simulation in this manner involves lengthy simulation times. The current approach to implement simulators as a Design for Manufacturing (DFM) solution has coupled these lengthy simulations with the need to expand the response calculations to greater areas of the device. The need for speed has driven the technology from software-only applications to specialty designed parallel-processing computer hardware capable of calculating feature imaging for the entire device layer. ${ }^{v}$ However, as a DFM solution, the only manufacturing-specific linkage that these advanced simulators present to "Lithography Aware Design" involves the investigation of five or six points within the measured Process Window of the target process to estimate the production boundaries of focus and dose variation for the simulation. This link results in a "Hot Spot" simulation that primarily calculates the probability of shorts or opens in the final printed image but 
also addresses the potential for CD size errors and variation, line-end pullback and the inclusion of "extra patterns" from printing of sub-resolution enhancement features. ${ }^{\text {vi }}$ The analysis does nothing to expand the process window itself.

In an industry that annually updates and changes techniques and technology, the current approach of DFM to Lithography Aware Design Verification solely employs as a link to manufacturing a classic technique called "Process Window" analysis that has existed in it's present form for over three decades. ${ }^{\text {vii }}$ Process Window calculations, historically used to estimate the initial focus and dose exposure variables for production setup, now are applied as the primary tool defining the exposure excursions anticipated in manufacturing. This is an application for which the process window technique was never designed and is certainly not optimized for.

Simulators attempt to enhance chip-design performance by focusing on the influence and optimization of pattern layout rather than design optimization. At no point does the design sequence incorporate an analysis of the manufacturing-process perturbation response for chip-structure design alternatives. Mask design alternatives that would result in more robust manufacturing performance and a concurrent reduction in the complexity of tool setup and process control.

Current process controls neglect the photomask as one of the largest sources of Across Chip Linewidth Variation (ACLV). Mask contribution is assumed stable throughout the process window ignoring the reality that it incorporates both static perturbation signatures, due to feature size offsets, as well as dynamic, optics-and-process sensitive signatures that vary with exposure as well as the unique aberrations of the exposure tool. ${ }^{\text {vii }}$

DFM techniques do not take into consideration the highly significant manufacturing conditions that vary continually during production such as the interaction of toolsets, process recipes and the incidence of production induced reticle haze. In essence, the complex response of aerial image interactions with the translucent wafer film stack presents a quantifiable influence that is strongly subjective to the final design selection of the OPC structure. This contribution cannot be derived from a simple process window calculation but is strongly reactive to the chip-design's stability to process fluctuations and must be tuned to the needs of each unique manufacturing process.

The industry needs a methodology that not only optimizes device-image fidelity for ideal processing conditions but can also expand the process awareness of the sequence to select mask-design alternatives that improve manufacturability.

\section{Proposed Solution by Process Behavioral Model Characterization}

In this study, a model-driven method of analysis of photoresist profiles is presented for several OPC mask-design alternatives without regard to the specific mechanics of the design itself. The design of each OPC feature is shown to be a strong contributor not only to resolution and ACLV but also to depth-of-focus and the stability of final image response; that is the ability of the feature profile to stay centered within the process window with a minimum of drift and variation.

Several different OPC designs for $70 \mathrm{~nm}$ features at half-pitch, 1:2 and 1:3 periodic loading are compared for their response stability to fluctuations of the process and the exposure tool perturbation signature set. Models are developed that allow the extraction of the nonlinear but systematic interactions of the designs with the normal fluctuations experienced across the process window plus those introduced by the toolset and film-stack.

Process Behavioral Models are presented as a method of extracting the systematic component of each feature's designiteration. These models provide the ability to quantify the specific response sensitivity of a design to systematic changes in the process films as well as drift introduced by the tools of the exposure set.

With this approach individual feature design alternatives can be comparatively characterized to allow selection of the final reticle-design-set that optimizes the breadth of the focus-dose process space and maximized manufacturability. The comparative analysis of various design options allows reticle design decisions to be made that enhance the reticle imaging for stability and robustness to process perturbations. Unlike simulation techniques, this method provides a customized analysis that considers the end-process's full process window plus the contributions from the unique toolset and film-stack variance. This method reduces the process-induced spread of feature variation about the design target and results in higher yields of high-performance final circuits. The resulting mask set will therefore reduce the need to customize exposure-tool and process setup for each unique device design. 
As a final point, the numbers we will present representing modeled variations across the exposure field are lower and more controlled than those experienced from statistical sampling. This is because the random noise and irrelevant process components present in the raw data have been removed. This methodology results in improved comparative performance with greater levels of confidence when evaluating the process capabilities of the chip design.

\section{PROBLEM FORMALIZATION}

\section{Experimental Setup}

A Focus-Exposure Dose matrix (FEM) was exposed using variations that approximate those seen in production. Dose was allowed to vary by $+/-3 \%$ and Focus by $+/-0.24$ um from tool nominal focus.

The experiment measured $70 \mathrm{~nm}$ target-size features generated using periodic targets with 1:1, 1:2 and 1:3, or isolated, feature patterns. As shown in Table 1, two design formats for patterns of each type were selected in both the Vertical ("V") and Horizontal (" $\mathrm{H}$ ") orientations to assist in determining the influence of scan on the response. All reticle structures included OPC correction with

\begin{tabular}{|l|l|l|}
\hline \multicolumn{1}{|c|}{ Design } & Orientation & Loading \\
\hline $\begin{array}{l}24 \mathrm{~B} 2 \\
54 \mathrm{~A}\end{array}$ & $\mathrm{~V} \& \mathrm{H}$ & $1: 1$ \\
\hline $\begin{array}{l}46 \mathrm{E} 4 \\
50 \mathrm{G} 4\end{array}$ & $\mathrm{~V} \& \mathrm{H}$ & $1: 2$ \\
\hline $\begin{array}{l}74 \mathrm{E} 6 \\
\text { 38F7 }\end{array}$ & V\&H & $1: 3$ \\
\hline
\end{tabular}

Table 1: Pattern designs and Characteristics. $\mathrm{V}=$ Vertical pattern edges, $\mathrm{H}=$ Horizontal assist features. To illustrate that intensive data metrology was not needed for the analysis, each exposure field contained only five sites for each feature type situated in the four corners and one in the chip interior.

All analyses presented are from one wafer exposure and one associated data set. The actual, physical variations in the design structures are not important for the object of this analysis and so are not presented.

A 0.75 Numeric Aperture, 193-Angstrom scanner was used for the exposure along

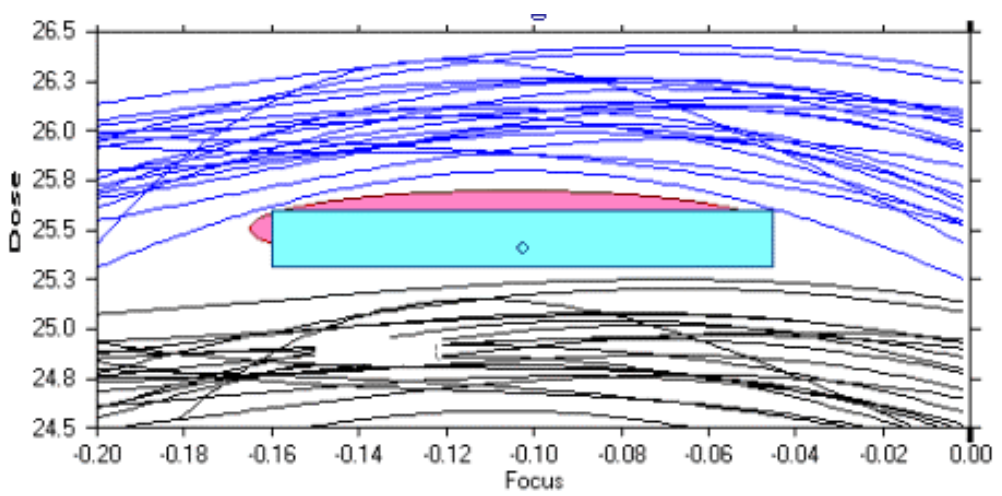

Figure 1: Process window for $70 \mathrm{~nm}$ patterns 74E6 and 38F7 with annular illumination. Wafer measurements were taken using a Nanometrics Scatterometer that employed OCD metrology with normal incidence, rotating polarized light (Nano 9030).

Model sequences, analysis and graphics for the study were generated using the commercial Weir PW software from TEA Systems. Data culling used the metrology tools quality indicator or mean square error (MSE) variable to remove soft data errors as well as automated culling and singularity-detection inherent in Weir PW during modeling.

\section{Concept of Design Alternatives}

A classic process window analysis performed on the features shows no remarkable variation due to feature design. The composite process window for the 1:3 feature loading patterns, shown in figure 1, exhibits a 0.112 micron (um) process Depth of Focus with only a $1.2 \%$ exposure latitude (EL\%). Since a manufacturing process window would like to see about $10 \%$ EL\% with 0.4 um DoF; even the "easy" lithography of the 1:3 patterns contains no mutually acceptable region. The Process Window methodology yields no clues as to what the limiting factors may be and a window of any size, which could encompass all six feature sets, does not exist by this method. Suggestions for design modification could not be gleaned from the classic Process Window analysis.

Many paths are available when mask feature design alternatives are considered. The primary reason for simulation of the photoresist image, given reticle pattern layouts, is the fact that "What you see" is not "What you get". Patterns lose the high frequency components of edges and corners. Sub-resolution enhancement features do not print under optimum exposure conditions and yet they continue to perturb the behavior of the final image. 


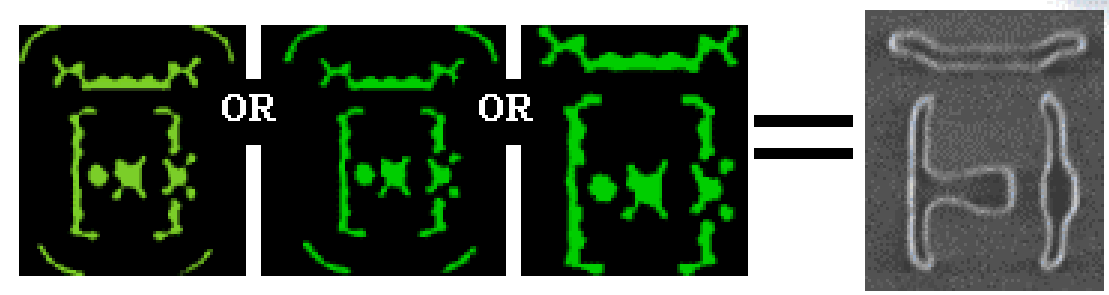

Figure 2: Three design alternatives for an SRAM Gate Binary Mask Pattern

Design, simulation and wafer image courtesy of Luminescent Technologies,

http://www.Luminescent.com

An easily visualized example of the alternatives available to chip design is regularly employed in the Inverse Lithography Technology of Luminescent. Unlike classic OPC driven simulations, the Luminescent simulator begins with the desired final image of the wafer and simulates the ray-trace back through the optical system of the scanner in reverse order. For "low k1" applications, this results in patterns that only vaguely resemble their final image on the wafer as shown in figure 2. At the optimum focus and exposure-dose, any of these mask-patterns will result in the desired wafer image however the final image response to variations in the process, that is their process windows, will differ. Depending upon the end process, mask manufacturer and scanner toolset, this difference can be very significant.

Design alternatives therefore provide an opportunity to optimize the process performance of each class of mask pattern and establish a customized set of guidelines for the device fabrication process. The optimal design format identified for a unique process can then be applied to each new mask design after the end-user's process has been characterized at least one time for the technology design rules and recipe of the device.

\section{Process Behavior Formalization}

At this point the classic Process Window algorithms are left behind in favor of a set of models that consider both the exposure and process perturbations that encompass the exposure process space. It's been shown that process disturbances are primarily systematic in nature and can be described as a relation of the form: ${ }^{3}$

$$
F R_{a}=\Lambda_{a} * D_{m}
$$

Where FRa is the feature response for pattern structure "a" that is the product of a series of process disturbances, " $\mathrm{m}$ " and their sensitivity coefficients " $\Lambda \mathbf{a}$ ". The "Feature Response" in this application can be any metrology profile or film feature with any "unit-of-measure". In patterning across the process space of our focus-dose matrix, the disturbances consist of the discrete variations of exposure-dose and focus in convolution with the scanner's optical train aberration response to defocus.

For this discussion we'll define exposure as the local focus and energy applied to the image field and the dose will refer to the energy imparted into the exposure. The spatial response of the pattern can therefore be described as:

$$
F R_{a}(x, y, f, d)=I F_{p}(x, y, f, d)+W_{p}(x, y)+r
$$

The feature response is a function of the feature's location on the field $(\mathrm{x}, \mathrm{y})$ resulting from the optic-distortion signature convolved with the perturbations of the photomask. This application, in crossing the focus-dose process space, will also exhibit the image's corresponding response to the exposure conditions set by the field's discrete offset of deFocus and dose $(\mathrm{f}, \mathrm{d})$. These perturbations vary in a systematic but asymmetric manner about the optimum exposure depending upon the aberrations present in the optical train.

Local variations in process, such as film thickness and reflectivity, contribute to a slowly changing systematic component that varies systematically across the wafer and is described by the $\mathrm{Wp}$ contribution. ${ }^{\mathrm{ix}}$ Variations arising from the $\mathrm{Wp}$ component across any single field of exposure, in this application, will be small relative to the intraField variation seen in moving from field-to-field due to the focus and dose changes of the focus matrix. 
The residuals to the perturbations will be random and are contained in the variable "r".

The first step in the analysis-sequence is to model the feature perturbations across each exposure field using an equation of the form:

$$
F R_{a}(r, \theta)=\beta_{o}+\sum_{j=1}^{n} \beta_{j} * r^{j} *(\cos (j \theta) * \sin (j \theta))+R_{a}
$$

Equation 3 describes the perturbations across the field as a function of the radial and angular $(\theta)$ position on a given exposure (f,d). Cylindrical coordinates are used in this example but rectangular coordinates can be substituted for a scanneroptimized model if a sufficient number of field points are measured across a regular array framework. The residuals to equation 3, embodied in Ra, will contain the wafer contributed systematic errors as well as the random errors of the data.

We define the model set describing feature performance for a given feature design as the set of Process Behavioral Models or simply it's "Process Model". Unlike the simple, regression-based, least squares models of trend fitting, the models of this form need to exhibit a set of characteristics that ensure a proper fit to the physical perturbations of the process and toolset. More specifically, the model must exhibit:

- Low coefficient covariance

- Extensive data culling capabilities for:

○ Gross metrology errors,

- Site-acquisition errors and

- Soft metrology errors.

- Data singularities during model fitting and

- A multi-level sequence of analysis that allows the systematic perturbations of one source to be extracted from the data before passing it on the to next analysis step.

Using this model the response of any feature design to a given set of exposure conditions can be quantitatively described by the offset coefficient of equation 3 ( $\beta \mathrm{o}$ ) with the corresponding response of the feature to the exposure tool and mask perturbations encountered at this exposure embodied in the summed coefficients $\mathrm{j}$ to $\mathrm{n}$. The offset coefficient will follow the classic Bossung response elements of a first-order variation with dose and a second order variation to the exposure's deFocus. Derivation of the process window elements of optimum focus, dose, Depth-of-Focus (DoF) and Exposure Latitude (EL\%) from this point onward is straight-forward and can be precisely tuned for basic exposure design-response as well a performance stability to deFocus and dose errors.

\section{MEASURING OVERALL DESIGN ROBUSTNESS}

\section{New Information from Classic Bossung Curves}

In applying the perturbation models of equation 3 and then extending the offset coefficients to a Bossung analysis we generate the first descriptive analysis of feature response to focus as shown in figure 3. At first glance this appears to be a classic Bossung deFocus graph but the information contained here is more representative of device response than a simple plot against focus would embody. The analysis also has eliminated the wafer-based contribution and can directly measure the sensitivity of the feature-design to across-chip aberrations for each exposure.

Each data point plotted in the graph of figure 3 represents the modeled response offset of the full-field at that exposure. The "error bars" of the data point detail the exposure's systematic Across Chip Linewidth Variation (ACLV) yielded by the structure design. Our confidence level for reading a true feature response is greater with this method because the modeling process enables the accurate removal of soft errors embedded in the data by the metrology as well as the stochastic perturbations of the process.

All of the features measured in the data of Figure 3 were intended for a $70 \mathrm{~nm}$ final size on the wafer. The chart as shown 
illustrates the now obvious offset of the dense-packed 1:1 features from those of the 1:2 and 1:3 groupings. The $25 \mathrm{mj} / \mathrm{cm} 2$ dose used to generate the data of this graphic apparently results in oversize $1: 1$ and undersized 1:2 and 1:3 loaded structures. However, there are additional offsets that were unobvious to the original device designer in the form of inherent response offsets between the natural feature size for the 1:2 and essentially isolated 1:3 loaded features.

Figure 3 also illustrates the near-isoFocal response of the 1:2 feature set contrasted against a 1:3 feature design that quickly deviates and falls off in size above 0.10 um defocus (38F7). The basic 1:1 feature design is continually changing with focus suggesting a pattern density and size that may be beyond the capabilities of this process and exposure tool set.

ACLV, shown by the error bars, varies significantly for the 1:3 data points where it appears to drastically rise out of control starting at 0.1 um deFocus. This would imply a drastically greater limitation in process space than was suggested by the Process Window of figure 1.

One final characteristic of the design shows a well-defined 3 to $4 \mathrm{~nm}$ offset in response size between the vertical and horizontal oriented features for the 1:1 and 1:2 loaded features. This is an artifact of the averaging of the lens-slit aberrations present in only one axis-oriented edge set of the feature. Proper design selection may be able to remove this.

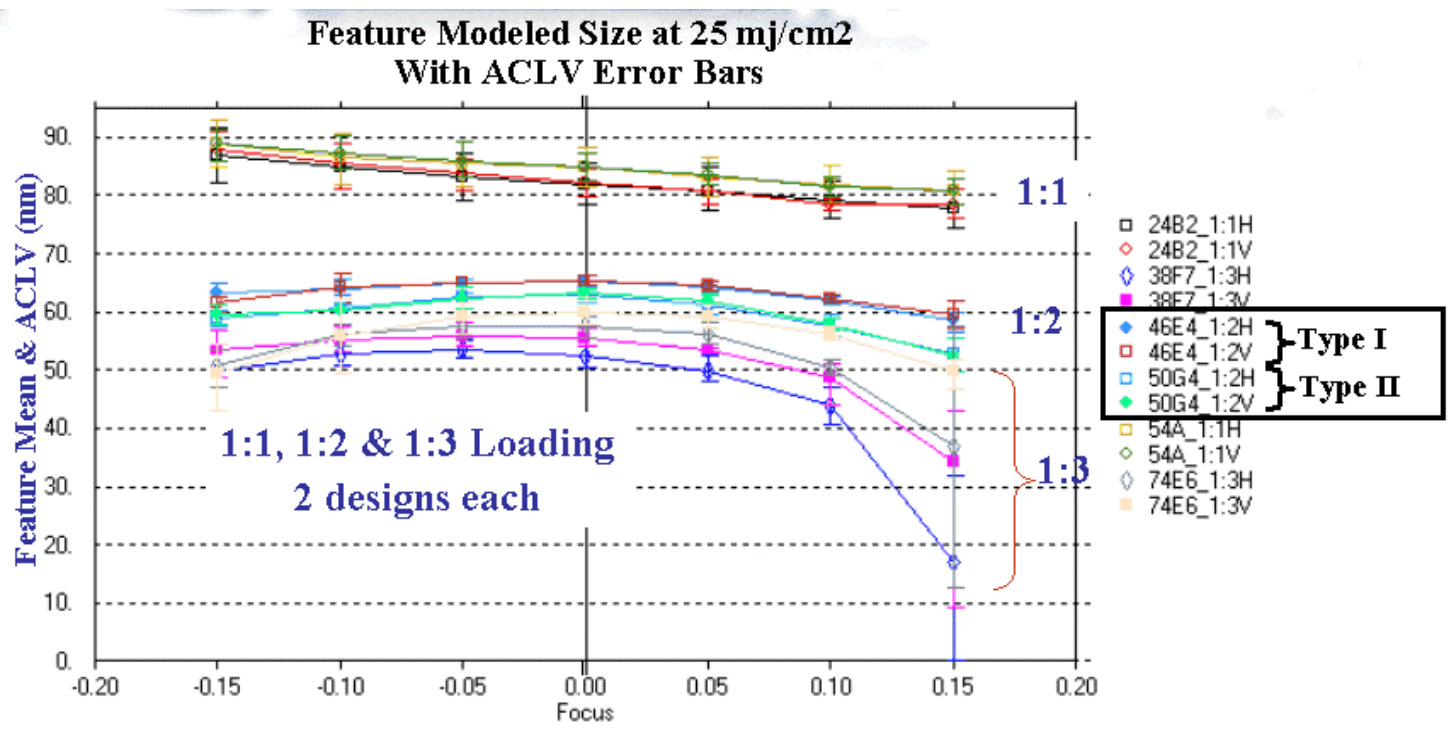

Figure 3: OPC Family response to focus with ACLV sensitivity error bars at optimum dose 


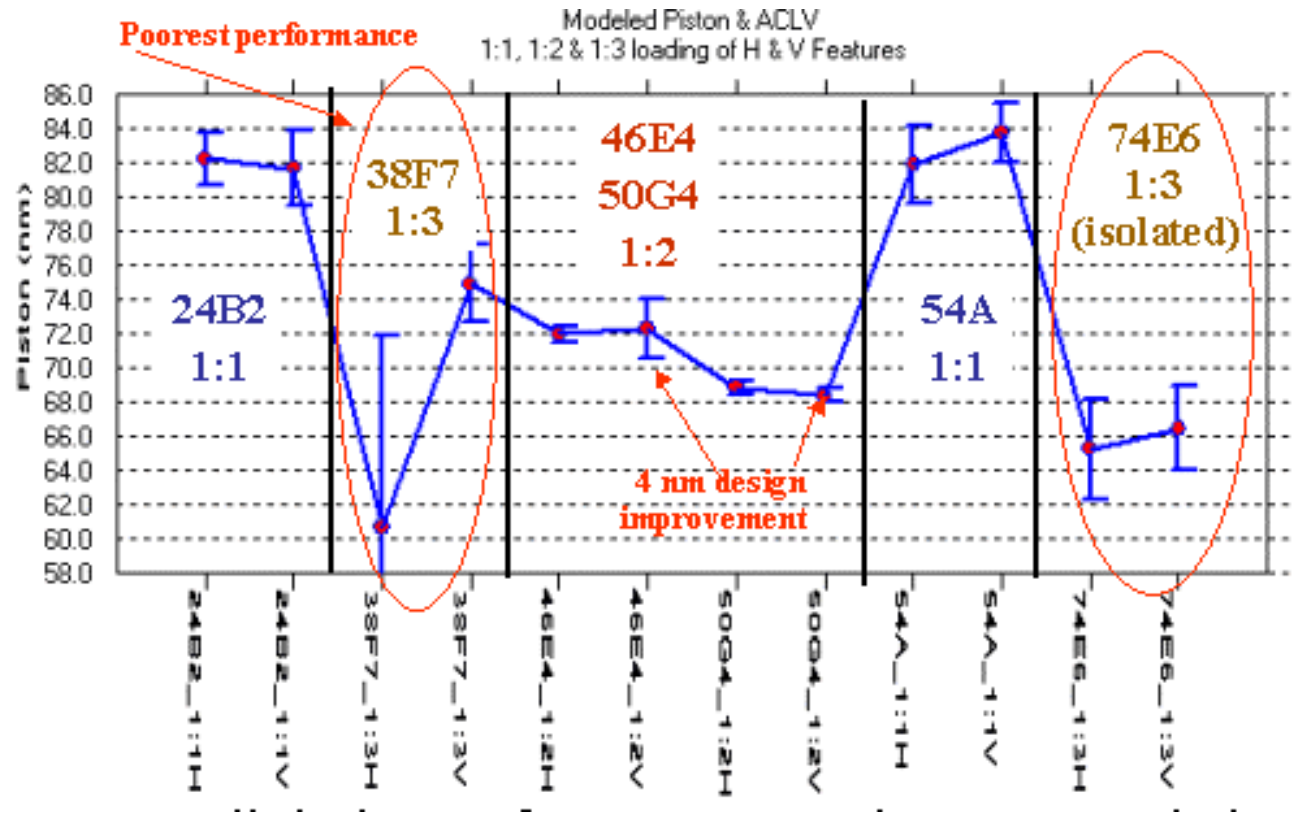

Figure 4: Full Process Space performance of four designs with modeled size and ACLV for the process-space

\section{Overall Process Response}

A comparison of the feature-design response across the perturbation spectrum of the process is shown in figure 4 as the natural values attained by each feature and it's associated range of systematic variation across the field for all exposure conditions. The 1:3 loaded feature sets exhibit the greatest variation in both the natural feature offset and their associated range due to process variations and aberration response. The strong offset in variation between vertical and horizontal features for the $38 \mathrm{~F} 7$ design is totally unacceptable as is the horizontal feature ACLV range. Comparing performance between these two "38F7" features, their ACLV range and offset, and the fact that the Vertical feature size more closely follows the 1:2 loaded features, we must conclude that the Horizontal feature design is at fault. The 74E6 designs, on the right side of the chart, are performing equally well with ranges in variation of about $5 \mathrm{~nm}$ total and a $1 \mathrm{~nm}$ difference in size between orientations $(\mathrm{V} \& \mathrm{H})$.

The best performance for this process is presented by the 50G4 and 46E4 designs for 1:2 loading. A $3 \mathrm{~nm}$ offset between designs base response illustrates that the nominal feature size can be closely adjusted at the design phase. The vertical features of the 46E4 design exhibit about $3 \mathrm{~nm}$ more ACLV than that of the horizontal suggesting a sensitivity of the design to reticle scan direction.

A process engineer employing the structures used in this experiment would therefore select the 50G4 design specifics for 1:2 feature loading along with the 1:3 features generated by the 74E6 structure that just marginally meets the ACLV specifications set out by the ITRS roadmap. A chip-designer should closely examine the design constraints of each feature

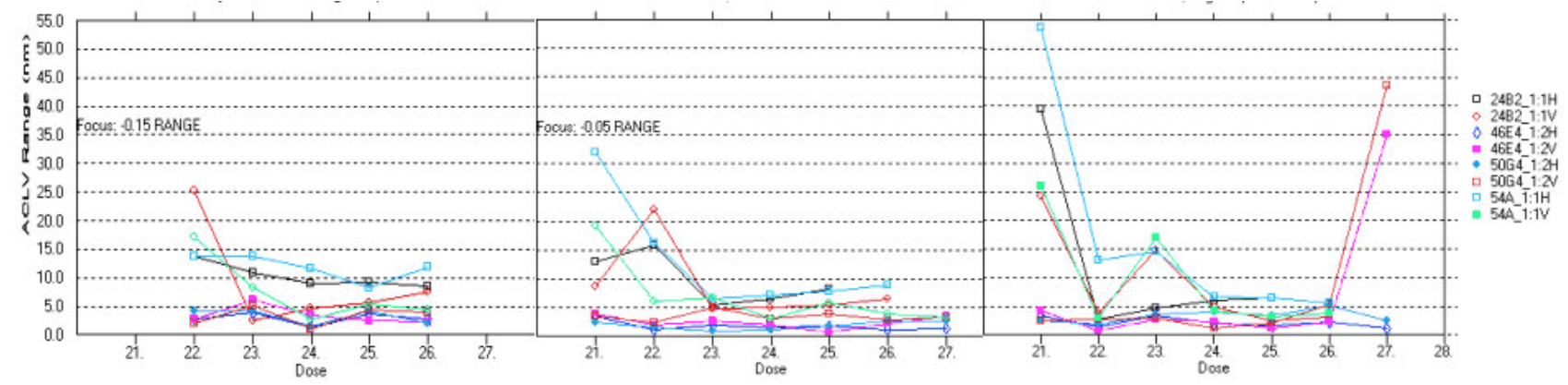

Figure 5: ACLV Response with focus and dose by design for $-\mathbf{0 . 1 5}$ um (left), $-\mathbf{- 0 . 0 5}$ um (center) and +0.1 um (right) defocus 


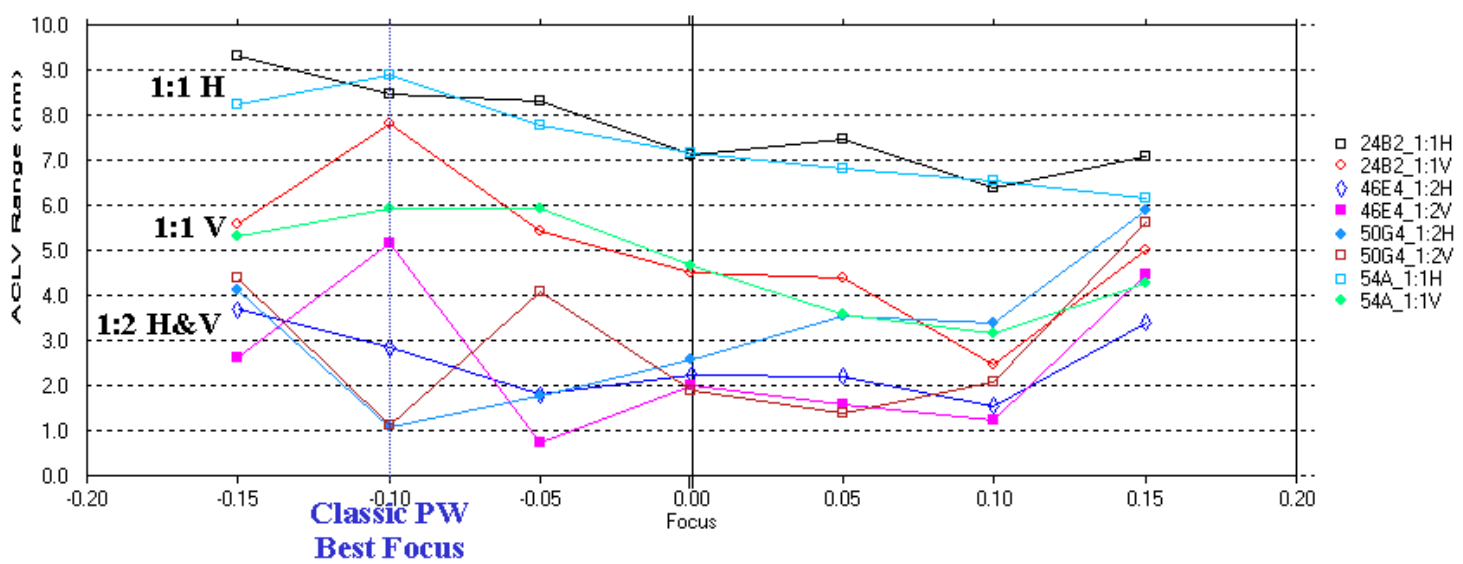

Figure 6: ACLV design response at $25 \mathrm{mj}$ for varying defocus of 1:1 and 1:2 loaded $70 \mathrm{~nm}$ features

family to determine the pattern options that would provide even greater process stability in future releases.

\section{Asymmetric ACLV Dose Response}

Feature uniformity across the exposure field changes as the feature-design attempts to compensate for changing dose and non-optimum focus conditions. Modeled ACLV ranges for the 1;1 and 1:2 loaded features are shown in figure 5.

The Classic Process Window of figure 1 calculated optimum focus to be located a -0.10 micron while the focus curves of figure 3 suggest a deFocus value of $-0.05 \mathrm{um}$. On the same graph, the optimum dose for feature-size is at $25 \mathrm{mj} / \mathrm{cm} 2$. An analysis of figure 5 shows that the exposure latitude of viable images at -0.15 um deFocus, now located only $-50 \mathrm{~nm}$ below the optimum classic focus, are severely restricted in Exposure Latitude. Under the same exposure conditions, the 1:1 vertical 24B2 feature design (red curve) quickly rises out of control with a $25 \mathrm{~nm} \mathrm{ACLV} \mathrm{at} 22 \mathrm{mj} / \mathrm{cm} 2$ dose.

Vertical feature designs for 1:1 features at this defocus setting all exhibit a larger ACLV of 10 to $15 \mathrm{~nm}$.

The +0.1 deFocus graph on the right side of figure 5 suggests the greatest range of exposure latitude and lowest ACLV could be gained for 1:1 and 1:2 loaded features if operations are performed closer to +0.1 um deFocus.

We next examine the detailed focus response of the 1:1 and 1:2 loading designs at the $25 \mathrm{mj} / \mathrm{cm} 2$ level using the graph of figure 6. This figure shows a well defined sensitivity to scan or slit direction in the 1:1, $70 \mathrm{~nm}$ feature designs with a reduction in ACLV as deFocus is moved from the Process Window "optimum" of -0.1 um up to $+0.1 \mathrm{um}$.

The 1:1 loading designs also exhibit a $3 \mathrm{~nm}$ ACLV increase with features oriented with their edges parallel to the direction of the slit (Horizontal features) suggesting a stronger sensitivity to reticle scan direction. The 1:2 loaded features show this sensitivity only when deFocus is greater than zero and then the ACLV increase is only $1 \mathrm{~nm}$.

The analysis tells us that operational ACLV could be minimized by setting nominal focus to approximately +0.05 as opposed to the -0.1 um deFocus suggested by the Classic Process Window Analysis. Design analyses also suggests that the 46E4 design for 1:2 loaded features performs better than the others for uniformity and robustness, shown in Figure 6 as ACLV-curve flatness with focus variation. 


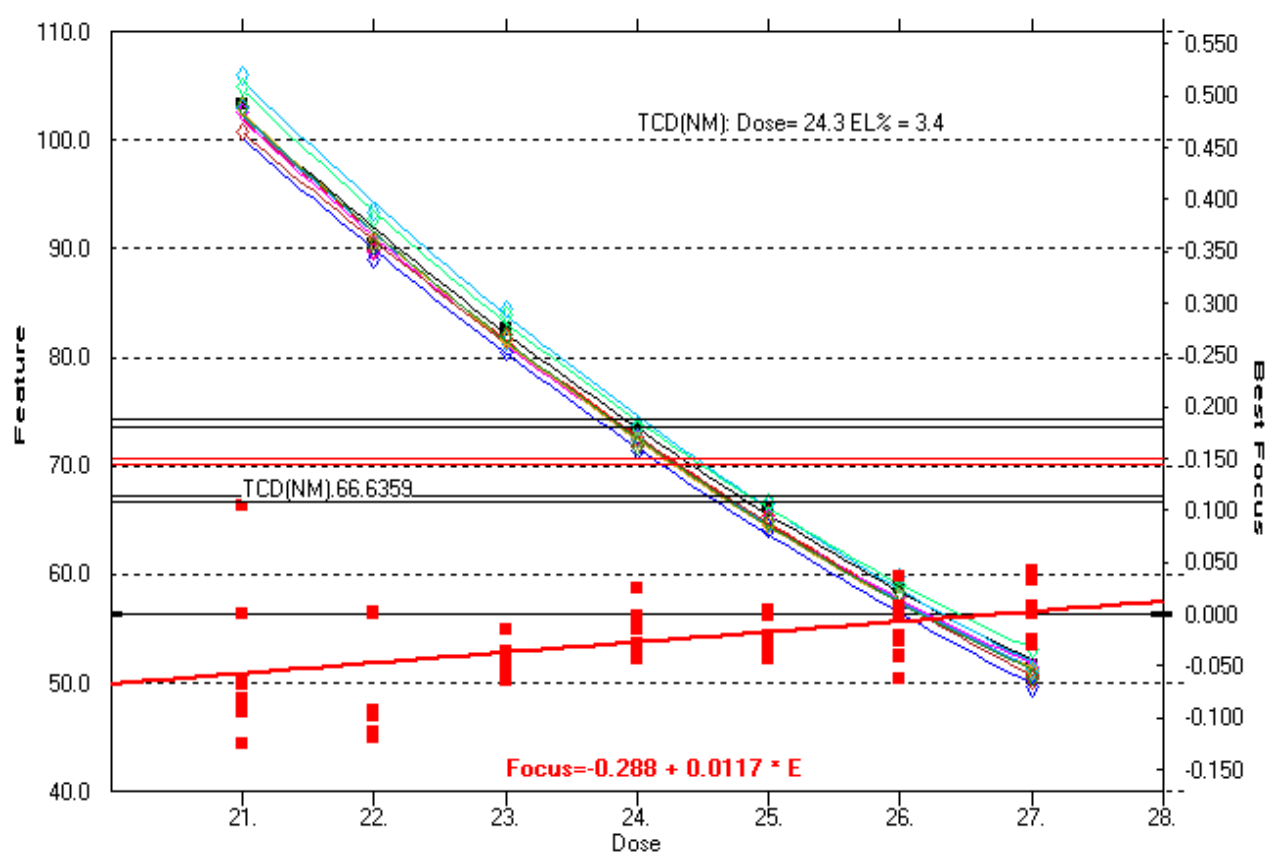

Figure 7: Dose and Best Focus response of 46E4, $70 \mathrm{~nm}$ 1:2 feature set after IntraField focus errors are removed.

\section{PROCESS EXHIBITED UNIFORMITY FROM DESIGN}

Having an understanding of the response characteristics of each device-structure we can next evaluate their respective process capability. The feature size-to-dose response exhibited by all the design sites across an exposure field can be evaluated after having removed the relatively strong size perturbations caused by local field focus errors as is shown in figure 7. ${ }^{\mathrm{x}}$ This technique yields a high confidence level in the true dose response of each field site for the dose-spectrum and always presents a feature-response curve with a high first-order dependency on dose. Variations from linearity on this curve are the result of Intra-Wafer perturbations and can also be removed if significant.

When aberrations are present, then "Best Focus" values for the feature change with dose. Best Focus values, and a first order trend line for the calculated Best Focus associated with each dose setting, are plotted at the bottom of figure 7. Best Focus values are scaled against the second ordinate $(\mathrm{Y})$ axis on the right side of the chart.

The Best Focus curve yields two important pieces of information. First the focus variation or data-spread across the field minimizes in the range of 23 to $25 \mathrm{mj} / \mathrm{cm} 2$. This is another indicator of the optimal exposure conditions for the feature set. Second, the Focus-to-Dose trend is linear and near zero in slope across the dose range indicating a system with small optical aberrations and well resolved features. The onset of aberrations from haze or lens-contamination will result in both a loss of focus uniformity and a strong nonlinear response of the optimum focus for each dose.

Figure 8 summarizes the Best Focus and modeled across-field focus variation exhibited by each 70 structure for low-k1 performance. Here we see equal focus response for the two orientations of the $46 \mathrm{E} 4$ feature with slightly lower focus uniformity for the horizontal structure. The $50 \mathrm{G} 4$ structure exhibits over $170 \mathrm{~nm}$ focus offset between horizontal and vertical orientations of the design. Horizontal focus variation about the optimum across the field is equal for both target designs suggesting that this may be more an artifact of the scanner than the design.

An examination of the spread in optimum dose values as they cross the target feature size of $70 \mathrm{~nm}$ in figure 8 yields a measure of the dose uniformity of exposure across the field. Dose uniformity here is a function of: 
- the individual structure fabricationoffsets on the photomask,

- process film variations across the photomask particularly for Phase masks,

- illumination uniformity across the reticle,

- reticle scan speed uniformity during exposure and

- feature-perturbation response to localized field aberration levels.

The across-field dose-uniformity required to reach a $70 \mathrm{~nm}$ target size for this process and reticle is shown in the graph of figure 9 for both design formats. The uncertainty in this dose calculation is $+/-0.005 \mathrm{mj} / \mathrm{cm} 2$. The 46E4 design exhibits a $0.06 \mathrm{mj} / \mathrm{cm} 2$ offset in

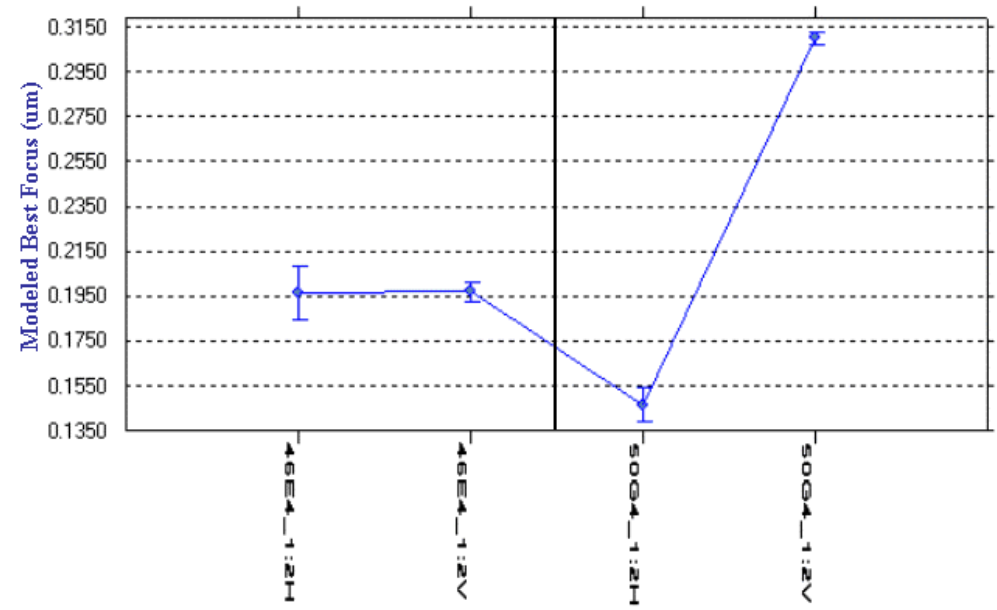

Figure 8: Best Focus exhibited by each feature and the variation, or focus-uniformity, modeled across the field as "error-bars" optimum dose with near equal across-chip variation. The contour plot for the vertical orientation of this structure is shown in the upper right of figure 9. Only five points across the field were used to generate this contour but the form is known from previous, more extensive data to be correct for this scanner. It is not uncommon to see a $1 \mathrm{mj} / \mathrm{cm} 2$ required optimum-dose uniformity on some currenttechnology, sub-65 $\mathrm{nm}$ reticles.

\section{Concept Application to Other Process Metrics}

Final feature uniformity in the form of etched or film-deposited structures is dependent upon the full edge-profile of small features. Optical Critical Dimension and CD-SEMS both are capable of including Side-Wall-Angle (SWA) measurements within the metrology dataset with little or no additional time expenditure. In spite of this technology, stepper engineers are still seen presenting arrays of SEM profiles of feature profiles to evaluate lens imaging across the process space.

SWA values are highly sensitive to focus and the level of sensitivity is governed by the degree of exposure biasing of the mask features. Process Modeled SWA variation across process space for our 1:2 loaded, $70 \mathrm{~nm}$ structures can be seen in figure 10.
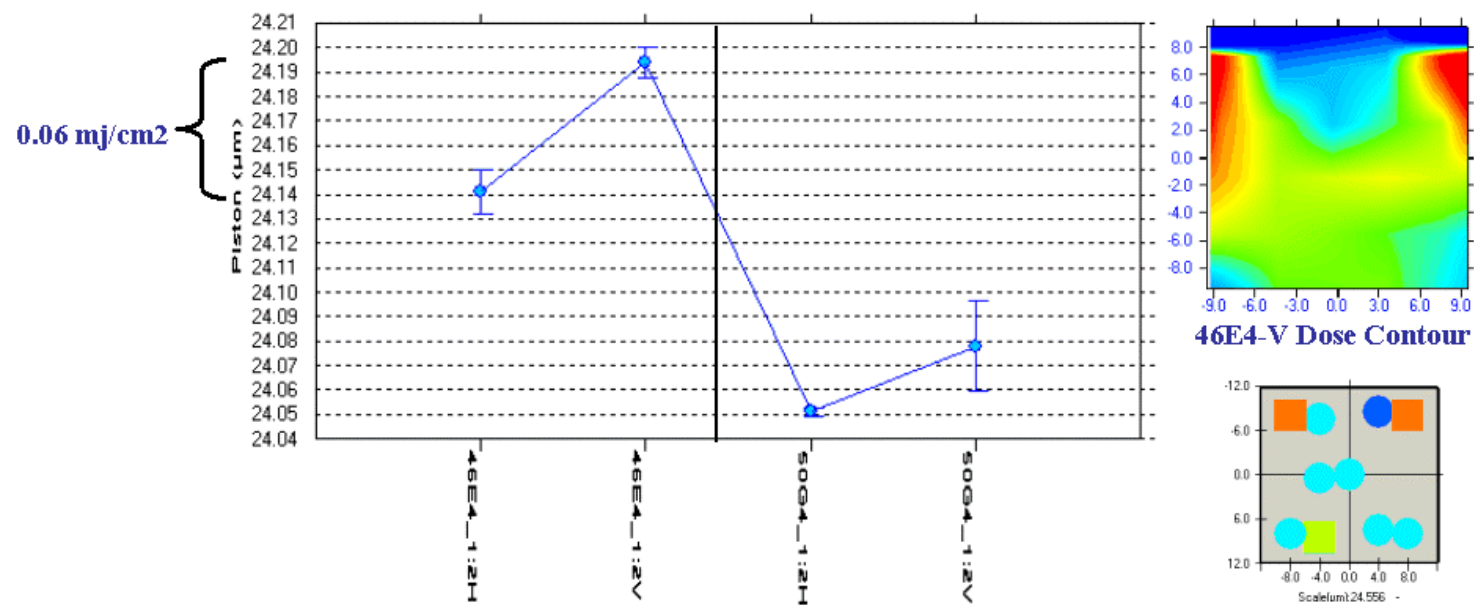

Figure 9: Modeled dose $\&$ uniformity error bars for 1:2 loading of 70 features, two designs and two orientations

Upper Right: Contour plot of vertical feature dose uniformity

Lower Right: Vector plot of locations and "dose-for-size" for both Vertical and Horizontal $46 E 4$ features 
The 46E4 nominal values are strongly centered about an ideal SWA value 86.1 degrees. The horizontal features exhibit less variation in this structure than the vertical features. The 50G4 design on the other had shows a small offset in nominal angle with almost equal variation across the process space. In this instance, the performance levels of SWA do not suggest a design advantage of either alternative.

The after-develop photoresist thickness across the process-space exposed field is modeled and the results are shown in figure 11. The obvious inability of the 1:1 features to properly image is shown by the large variation and relatively thick remaining modeled photoresist. Earlier results suggested the 46E4, 1:2 loaded feature design as generating superior performance. Figure 11 here shows the stability of energy coupling into the photoresist for the $46 \mathrm{E} 4$ feature whose final thickness is within $3 \mathrm{~nm}$ of the 1:3 loaded structures. Photoresist thickness measured and modeled in this manner may be a rapid gage for quality evaluation of future feature designs but that's a topic for later studies.

\section{CONCLUSIONS}

The current "Design for Manufacture" approach of the industry is very complex. Photomask file-sizes and workloads spiral while users attempt to conjure imaging schemes that favor specific feature designs. Even the eventual adoption of a "Double Patterning" solution to proximity problems only promises to temporarily stall the complexity trend spiral while simultaneously increasing both photomask and overlay burdens.

Process-tuning complexity is projected to increase to even greater heights through a combination of end-user directed

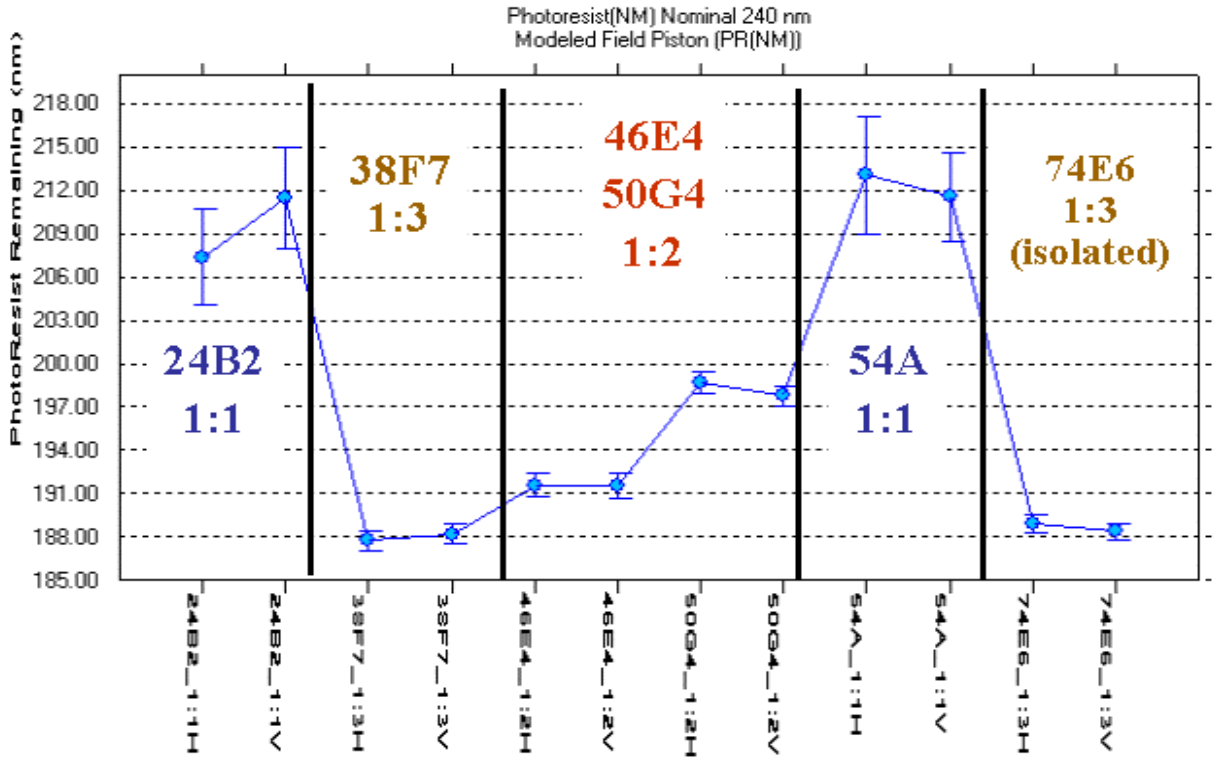

Figure 11: A nominal photoresist thickness of $240 \mathrm{~nm}$, modeled from data after develop for each device design.

Plotted data shows the measured photoresist thickness over the 50 um target site. Error-bars for each site contrast the variation in thickness of the feature's film across the full process space and exposure field. 
techniques that attempt to adjust the process to the design rather than have the design consider alternatives that would increase the tolerance of the final image to process variations.

Simulators used for mask pattern design currently drive toward the resolution of the features with little regard for processspace variations in exposure, film uniformity and optical capabilities. Even the incorporation of classic Process Window links in the simulation does little more than search for shorts, opens and gross feature-size variations. Process tolerance of the design and the unique capabilities of the device manufacturer are not considered attributes during simulation.

A simpler and more cost effective approach to optimized design selection has been presented in techniques that can compare the performance of critical-feature design alternatives and model their robustness to a user's unique capabilities. This ability to generate process and design-specific Process Behavioral Models provides the opportunity to evaluate the full spectrum of design alternatives, photomask-manufacturing signatures and device-manufacture process capabilities to select an optimum set of patterns from the basic design alternatives of the reticle. The result is a photomask that works best for each specific process and exhibits superior tolerance to process variations while minimizing the hassle involved in the complex techniques currently used to control illumination characteristics on the exposure tool.

This analysis has shown how several simple variants in OPC design decisions can generate widely varying feature size and ACLV response across the process space. These results cannot be derived using the classic Process Window analysis approach but their existence defines the difference between failure and success in the early stages of manufacture.

This technique involves the application of Process Behavioral Models to match the optimum device design alternatives to the needs of a specific device process rather than tune each process to the design. These models provide an intelligent and customized approach to the early selection of process-optimized design alternatives and their use will result in improved manufacturing yields that are achieved earlier in each new-product ramp.

\section{REFERENCES}

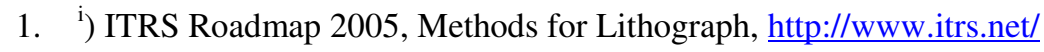

2. ii) Mircea Dusa, Richard Moerman, Bhanwar Singh, Paul Friedberg, Ray Hoobler, Terrence Zavecz, "Intra-wafer CDU characterization to determine process and focus contributions based on Scatterometry Metrology", Proc. SPIE (2004), Vol. 5378-11.

3. iii) Jun Zhu, et. al., "A Study of Double Exposure Process Design with Balanced Performance Parameters for Line/ Space Applications", Fuji-Film Interface November 2006.

4. iv) A. Balinski, L.Karklin, V.Axelrad, "Integrated Scheme for Semiconductor device verification", US Patent Office 6681376, Oct. 17, 2001

5. ") Yu Cao, Yen-Wen Lu, Luoqi Chen and Jun Ye, "Optimized Hardware and Software for Fast, Full Chip Simulation", SPIE ( 2005) vol. 5754_40.

6. ${ }^{\text {vi }}$ ) C. Lin, M. Tsai, T. Chen, T.Wang, Y.Chang, "Recent Research and Emerging Challenges in Physical Design for Manufacturing/ Reliability", National Science Council of Taiwan, 2007 Grant \# NSC 95-2221-E-002-372

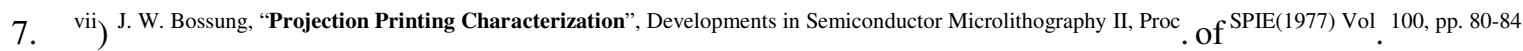

8. viii) T. Zavecz, "Full sub-65 nm data-modeling for Photomask Manufacturing", FujiFilm Interface 2006, November 2006

9. ${ }^{\text {ix }}$ ) Mircea Dusaa, Richard Moermanb, Bhanwar Singhc, Paul Friedbergc, Ray Hooblerd, Terrence Zavecz, " Intra-wafer CDU characterization to determine process and focus contributions based on Scatterometry Metrology", SPIE (2005) vol. 5378_11.

10. ${ }^{x}$ ) T. Zavecz, "Bossung Curves; an old technique with a new twist for sub-90 nm nodes", SPIE (2006) vol. 6152 - 109 